Pacific Journal of Mathematics

THE INDEX OF A TANGENT 2-FIELD 


\section{THE INDEX OF A TANGENT 2-FIELD}

\section{Mark Mahowald}

Thomas, using an obstruction theory approach, evaluated the index of a tangent 2-field on $M^{m}, m \equiv 1(4)$ if $M$ is a spin manifold. Atiyah using the Atiyah-Singer index theorem evaluated the index for all orientable manifolds. The purpose here is to give a proof of Atiyah's result in the spirit of Thomas' work.

Let $M$ be a connected closed smooth orientable manifold of dimension $m$. Let $k$ be any integer and suppose $M$ admits $k$ vector fields which are linearly independent everywhere except possibly at a finite number of points. The obstruction to making the $k$ vector field linearly independent everywhere is called the index of the $k$-field and it is an element of

$$
H^{m}\left(M, \pi_{m-1}\left(V_{m, k}\right)\right) \simeq \pi_{m-1}\left(V_{m, k}\right)
$$

Suppose $m=2 r+1$ and let

$$
\hat{\chi}_{2}(M)=\left(\operatorname{dim} \bigoplus_{i=0}^{r} H^{i}\left(M, Z_{2}\right)\right) \bmod 2 . \quad \text { In [5], }
$$

Thomas proved:

THEOREM. Let $M$ be a closed connected spin manifold, $m \equiv 1(4)$, $m>1$ with $W_{m-1}(M)=0$. Then the index of any 2-field with singularities is

$$
\hat{\chi}_{2}(M) \in Z_{2}=\pi_{m-1}\left(V_{m, 2}\right) .
$$

Thomas' method was to calculate the secondary obstruction to a cross section of the association $V_{m, 2}$ bundle to the tangent bundle. Atiyah [1] showed that if

$$
b=\left(\operatorname{dim} \bigoplus_{i=0}^{r} H^{i}(M, \text { Reals })\right) \bmod 2
$$

then the index of a 2-field for any orientable manifold with $W_{m-1}(M)=0$ is $b$. Finally, Milnor, Lusztig, and Peterson [3] showed the relationship between these results by showing that 


$$
b+\hat{\chi}_{2}=W_{2} W_{m-2} \text {. }
$$

It has always seemed that direct proof, in the spirit of Thomas, should be possible for the Atiyah result. In this paper we will provide such a proof, i.e., we will prove

THEOREM 1. Let $M$ be a closed connected orientable manifold $m \equiv 1(4), m>1$ with $W_{m-1}(M)=0$. Then the index of any 2-field with finite singularities is

$$
\left(\hat{\chi}_{2}+W_{2} W_{m-2}\right) \in Z_{2}=\pi_{m-1}\left(V_{m, 2}\right) .
$$

2. Proof of the theorem. The proof has two key steps. The first is to show that a secondary operation on the Thom class involves the secondary obstruction; and the second step is to evaluate the cohomology operation.

Let $m \equiv 1 \bmod 4$. Then

$$
S q^{2} S q^{m-1}+S q^{m} S q^{1}=S q^{m+1}
$$

and, thus, on $m$-dimensional integral classes $S q^{2} S q^{m-1}=0$. Let $E$ be the fiber of the map

$$
K(Z, m) \stackrel{S q^{m-1}}{\longrightarrow} K\left(Z_{2}, 2 m-1\right) .
$$

Then the relation $S q^{2} S q^{m-1}=0$ defines a class $v \in H^{2 m}\left(E, Z_{2}\right)$ which is defined up to a primary operation on the generator of $H^{m}(E, Z)$.

THEOREM 2.1. Let $T(M)$ be the Thom complex of $\tau(M)$, where $M$ is a manifold as in Theorem 1. There exists a map $f: T(M) \rightarrow E$ such that $f^{*}$ in dimension $m$ is an isomorphism and $f^{*}(v)=$ $U \cup\left(\mathrm{O}_{2}+W_{2} W_{m-2}\right)$ where $\mathrm{O}_{2}$ is the index of the 2-field.

This is proved in [4].

THEOREM 2.2. For the data as in Theorem $2.1, f^{*}(v)=\hat{\chi}_{2}(U \cup \mu)$ where $\mu$ generates $H^{m}\left(M, Z_{2}\right)$.

This is the new result which we prove in $\$ 3$. The main theorem is a direct consequence of these two results.

3. Proof of Theorem 2.2. Recall that the tangent bundle embeds in a natural way as a neighborhood of the diagonal in $M \times M=$ 
$M^{2}$. Let $j: M^{2} \rightarrow T(\tau(M))$ be the obvious map. Let $\left\{\alpha_{i} ; i=1, \cdots, q\right\}$ a basis for

$$
\bigoplus_{j=0}^{(m-1) / 2} H^{j}\left(M, Z_{2}\right) \quad \text { and } \quad\{\beta\}
$$

be the dual basis, i.e.,

$$
\alpha_{i} \cup \beta_{j}=\delta_{i j} \mu .
$$

Proposition 3.1 [Theorem 2.6 [5]].

(a) $j^{*} U=A+t A$ where $A=\sum_{j=0}^{q}\left(\alpha_{i} \otimes \beta_{i}\right)$.

(b) $A \cup t A=\hat{\chi}_{2}(M) \mu \otimes \mu$.

Let $\tilde{\Omega}_{m}$ be the secondary operation defined over $K\left(Z_{2}, m\right)$ based on $S q^{2} S q^{m-1}+S q^{1}\left(S q^{m-1} S q^{1}\right)=0$.

Proposition 3.2. [Thomas 2.6 [5]]. If $S q^{m-1} U=0$ then $S q^{m-1} A=0$.

Proof. An easy application of the Cartan formula shows that $S q^{m-1} A \in H^{m-1}\left(M, Z_{2}\right) \otimes H^{m}\left(M, Z_{2}\right)$. Thus, $S q^{m-1} A$ and $S q^{m-1}(t A)$ are in different graded subgroups of $H^{*}\left(M^{2}\right)$ and so could add to zero only if each were zero separately.

Proposition 3.3. $S q^{m-1} S q^{1} A=\left\langle V_{r} \cup S q^{1} V_{r}[M]\right\rangle \mu \times \mu$ for any choice of basis $\alpha_{i}$ where $V_{r}$ is the $r$ dimensional $W u$ class.

Proof. Since $S q^{m-1} S q^{1} A=S q^{m-1} S q^{1}\left(\Sigma \alpha_{j} \otimes \beta_{j}\right) \quad\left(\operatorname{dim} \alpha_{j}=r\right)$ it suffices to verify that if $H^{r}\left(M, Z_{2}\right)$ is a vector space of rank $t$ and if $N$ is a linear transformation taking the $\alpha_{i}$ to the new basis $\bar{\alpha}_{i}$ then

$$
S q^{m-1} S q^{1}\left(\Sigma N \alpha_{j} \otimes N^{*} \beta_{i}\right)=S q^{m-1} S q^{1}\left(\Sigma \alpha_{j} \otimes \beta_{j}\right)
$$

Moreover, $N$ can be written as a composite of permutations (which obviously leave it invariant) and transformations of the form

$$
N_{i j} \alpha_{k}= \begin{cases}\alpha_{k} & k \neq j \\ \alpha_{i}+\alpha_{j} & k=j\end{cases}
$$

So the lemma is true if it is true for $N_{i j}$. Now $N_{i j}^{*} \beta_{k}=$ $\left\{\begin{array}{ll}\beta_{k} & k \neq i \\ \beta_{i}+\beta_{j} & k=i\end{array}\right.$. Thus the difference between the two sums is easily seen to be 0 . 
Now notice that $S q^{m-1} S q^{1}\left(\alpha_{i} \otimes \beta_{i}\right)=V_{r} S q^{1} \alpha_{i} \otimes V_{r} \beta_{i}$ and since $V_{r} S q^{1} \alpha_{i}=\left(S q^{1} V_{r}\right) \alpha_{i}$, if $S q^{1} V_{r}=0$ then the lemma is true. Assume then $S q^{1} V_{r} \neq 0$, and give a basis for $H^{r}\left(M, Z_{2}\right)$ by choosing $\alpha_{1}, \cdots, \alpha_{t-1}$ to span $\left\langle S q^{1} V_{r}\right\rangle^{\perp}$ and filling out to a basis by requiring $\alpha_{t}$ be dual to $S q^{1} V_{r}$. Then

$$
S q^{m-1} S q^{1}\left(\Sigma \alpha_{j} \otimes \beta_{j}\right)=S q^{1} V_{r} \alpha_{t} \otimes V_{r} S q^{1} v_{r}
$$

and the lemma follows.

Proposition 3.4. Theorem 2.2 is true if $S q^{m-1} S q^{1} A=0$, i.e., if $V_{r} \cup S q^{1} V_{r}=0$.

With the additional hypothesis that $W_{2}=0$ this is exactly what Thomas proved in [5]. The proof which follows is the same as Thomas' up to the point where it is shown that the indeterminancy does not kill the argument.

Proof of 3.4. Let $\left(E_{1}, u, v\right)$ be the universal example for $\tilde{\Omega}_{m}$, i.e., $E_{1}$ is a two stage Postnikov system with $k$-invariants $S q^{m-1}$ and $S q^{m-1} S q^{1}$ over a $K\left(Z_{2}, m\right)$. The class $u$ is the image of the fundamental class of $H^{m}\left(K\left(Z_{2}, m\right)\right)$ in $H^{m}\left(E_{1}\right)$. The class $v \in H^{2 m}\left(E_{1}\right)$ is defined by the relation $S q^{2} S q^{m}+S q^{1}\left(S q^{m-1} S q^{1}\right)=0$. The hypotheses imply that there is a commutative diagram

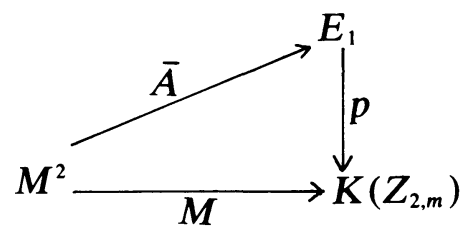

where $A^{*}(\kappa)=A$ and $\kappa_{m}$ is the fundamental class of $K\left(Z_{2, m}\right)$. Let $t \bar{A}$ be the composite $M^{2} \stackrel{t}{\rightarrow} M^{2} \stackrel{A}{\rightarrow} E$. Consider the diagram (not necessarily commutative)

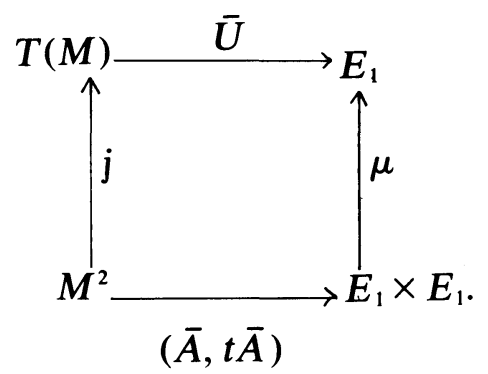

The argument which Thomas used goes as follows: First

$$
\mu *(v)=v \otimes 1+p *(\kappa \otimes \kappa)+1 \otimes v,
$$


since $v$ is not primitive, (see [5] or [2]). Then, since $(\bar{A}, t \bar{A}) *(v \otimes 1)=$ $(\bar{A}, t \bar{A})^{*}(1 \otimes v)$, we see that

$$
(\mu(\bar{A}, t \bar{A}))^{*} v=A \cup t A=\hat{\chi}_{2}(M)(\mu \otimes \mu) .
$$

Now $d(\mu(\bar{A}, t \bar{A}), \bar{U} j)$, the difference class, is a map into

$$
K\left(Z_{2}, 2 m-2\right) \times K\left(Z_{2}, 2 m-1\right)
$$

and thus is a pair of cohomology classes, $(a, b)$. It follows from the definition of the secondary operation that

$$
\left(\bar{U}_{j}\right)^{*} v=(\mu(\bar{A}, t \bar{A}))^{*} v+S q^{2} a+S q^{1} b .
$$

Since $M$ is orientable $S q^{1} b=0$ and since in Thomas' case $S q^{2} W_{2}(M)=$ $0, S q^{2} a=0$. What we need to show is that in the case of our diagram the same conclusion holds.

Lemma 3.5. Let $(a, b) \in H^{2 m-2}\left(M^{2}, Z_{2}\right) \otimes H^{2 m-1}\left(M^{2}, Z_{2}\right)$ be the pair of cohomology classes $(a, b)=d(v(\bar{A}, t \bar{A}), \bar{U} j)$. The class $a$ is invariant under $t^{*}$.

The proof is given in $\$ 4$. We continue the proof of 3.4.

Thus if $(a, b)=d(v(\bar{A}, t \bar{A}), j \bar{U})$ then $a$ is a symmetric class, i.e.,

$$
a=a_{1} \otimes \mu+a_{2} \otimes a_{2}+\mu \otimes a_{1} .
$$

Now $S q^{2} a=0$ if $a$ is symmetric; and, therefore, if we use the diagram* with the maps as given we see that

$$
(\bar{U} j) * v=\hat{\chi}_{2}(M)(\mu \otimes \mu) .
$$

This is 2.2 under the hypothesis of 3.4.

We now consider the case where $V_{r} \cup S q^{1} V_{r} \neq 0$. Let $A^{\prime}=$ $A-V_{r} \otimes S q^{1} V_{r}$. Then $j^{*} U=A^{\prime}+t A^{\prime}+S q^{1}\left(V_{r} \otimes V_{r}\right)$. Let $(E, u, v)$ be the universal example for the operation $\Omega$ based on the relation $S q^{2} S q^{m-1}=0$ which holds on integral classes. The class $u \in H^{m}(E, Z)$ is the fundamental class and $v \in H^{2 m}\left(E, Z_{2}\right)$ is based on the relation. Let $f: M^{2} \rightarrow E$ be such that $f^{*} u=A^{\prime}+t A^{\prime}$ and suppose $f=-t f$. Then $\Omega\left(A^{\prime}+t A^{\prime}\right)=(\hat{\chi}(M)-1)(\mu \otimes \mu)$. Note that $\Omega$ is also defined on $S q^{1}\left(V_{r} \otimes V_{r}\right)$. Let $E_{2}$ be the fiber of the map $K\left(Z_{2}, m-1\right)$ $\delta S q^{m-3}$

$\stackrel{\delta S q^{m-3}}{\longrightarrow} K(Z, 2 m-3)$. Let $u_{2}$ be the fundamental class. Suppose a map defining $\Omega$ on $S q^{1}\left(V_{r} \otimes V_{r}\right)$ factors $M^{2} \rightarrow E_{2} \leftrightarrow E$ where $g^{*} u=S q^{1} u_{2}$ and $k^{*} u_{2}=V_{r} \otimes V_{r}$. The indeterminancy of the value of $\Omega$ via such factorization is $k^{*}\left(S q^{2} H^{2 m-2}\left(E_{2}\right)\right)$ but it is easy to see that $H^{2 m-2}\left(E_{2}\right)$ is generated by primary operations on $u_{2}$ and primary operations on a 
symmetric class are symmetric and thus $k^{*}\left(S q^{2} H^{2 m-2}\left(E_{2}\right)\right)=0$. Thus to complete the proof of 2.2 we need to show that $k$ exists, (Lemma 3.6), and we need to evaluate $\Omega$ on such a factorization (Lemma 3.7).

LEMMA 3.6. $\delta S q^{m-3}\left(V_{r} \otimes V_{r}\right)=0$

Proof. Since $W_{m-1}(M)=0$ and $W_{m-2}(M)$ is the reduction of an integer class $\delta * W_{m-3}(M)$ we see that $W_{2 m-4}(M \otimes M)=$ $W_{m-2}(M) \otimes W_{m-2}(M)$ is the restriction of an integer class and so $\delta\left(W_{2 m-4}(M \otimes M)\right)=0$ but $\delta\left(W_{2 m-4}(M \otimes M)\right)=\delta S q^{m-3}\left(V_{r} \otimes V_{r}\right)$.

LEMMA 3.7. Let $c$ be a class of dimension $m-1$ with $\delta S q^{m-3} c=0$, where $\delta$ is the Bockstein $H^{*}\left(, Z_{2}\right) \rightarrow H^{*+1}(, Z)$. Then $(E, u, v)$ is defined on $S q^{1} c$ and equals $S q^{m-1} S q^{2} c$ modulo a primary operation on $S q^{1} c$.

This is proved in $\S 5$.

This finishes the proof since $S q^{m-1} S q^{2}\left(V_{r} \otimes V_{r}\right)=$ $S q^{r} S q^{1} V_{r} \otimes S q^{r} S q^{1} V_{r}$ and $S q^{r} S q^{1} V=S q^{2} S q^{r-1} V_{r}$. Now $S q^{r} S q^{1} V \neq 0$ iff $V_{r} \cup S q^{1} V_{r} \neq 0$ and iff $S q^{2} S q^{r-1} V_{r}=S q^{2} W_{m-2} \neq 0$ but $V_{2}=W_{2}$ and if $V_{r} \cup S q^{1} V_{r} \neq 0, S q^{m-1} S q^{2}\left(V_{r} \otimes V_{r}\right) \neq 0$ and $W_{2} W_{m-2} \neq 0$. This completes the proof.

4. Proof of 3.5. Let $\bar{E}$ be the fiber of the map $K\left(Z_{2}, m\right)-\underset{S q^{m-1}}{\rightarrow} K\left(Z_{2}, 2 m-1\right)$. Let $[X]^{k}$ be a $Z_{2}$ homology skeleton of the space $X$, i.e., $i^{*}: H^{j}\left(X, Z_{2}\right) \rightarrow H^{j}\left([X]^{k}, Z_{2}\right)$ is an isomophism for $j \leqq k$ and $H^{j}\left([X]^{k}, Z_{2}\right)=$ for $j>k$. Then

$$
\left[\left[M^{2} /\left[M^{2}\right]^{m-1}\right]^{2 m-1}, \bar{E}\right] \cong\left[\Sigma^{-2}\left(\left[M^{2} /\left[M^{2}\right]^{m-1}\right]^{2 m-2}\right), \Omega^{2} \bar{E}\right]
$$

and

$$
\left[M^{2} /\left[M^{2}\right]^{m-1}, \bar{E}\right] \cong\left[\left[M^{2} /\left[M^{2}\right]^{m-1}\right]^{2 m-2}, \bar{E}\right]
$$

Therefore

$$
\Sigma^{-2}\left[M^{2} /\left[M^{2}\right]^{m-1}\right] \Omega^{2} \bar{E} \cong\left[M^{2} /\left[M^{2}\right]^{m-1}, \bar{E}\right]=A .
$$

This isomophism is not canonical since it depends on the particular desuspension used. Suppose we choose one so that $j$ desuspends to

$$
j^{\prime}: \Sigma^{-2}\left(\left[M^{2} /\left[M^{2}\right]^{m-1}\right]\right)^{2 m-2} \rightarrow \Sigma^{-2}\left([T(M)]^{2 m-2}\right) .
$$

Since $\Omega^{2} \bar{E}=K\left(Z_{2}, m-2\right) \times K\left(Z_{2}, 2 m-4\right)$ we see that $A$ is isomorphic to some extension of $H^{m}\left(M^{2}, Z_{2}\right)$ by $H^{m-2}\left(M^{2}, Z_{2}\right)$. The extension is determined by the loop multiplication in $\Omega^{2} \bar{E}$.

The following lemma is an easy calculation. 
Lemma 4.1. For any class $a \in A$ represented by $\left(a_{1}, a_{2}\right)$ with $a_{1} \in H^{m}\left(M^{2}, Z_{2}\right), 2 a$ is represented by $\left(0, S q^{m-2} a_{1}\right)$.

Since $t^{*}$ on $\left(\operatorname{Imj} j^{*}\right)$ is fixed and since $t^{*} S q^{m-2} a=S q^{m-2} t^{*} a$, the subset in $\left(H^{m}\left(M^{2}, Z_{2}\right), H^{2 m-2}\left(M, Z_{2}\right)\right)$ consisting of classes which are invariant under $t^{*}$ is subgroup. Let $E_{1} \stackrel{p}{\rightarrow} \bar{E}$ be the natural projection. Clearly $j \bar{U} P$ and $(\bar{A}+t \bar{A}) P$ are maps in this subgroup and their difference is $a$ where $(a, b)=d(j \bar{U}, \bar{A}+t \bar{A})$. Hence, $t^{*} a=a$.

5. Proof of 3.7. We will need to study several two stage Postnikov systems simultaneously and so some additional notation is needed. Let $\beta$ be a vector of primary operations and $K(G)$ a generalized Eilenberg-MacLane space

$$
K(G)=\Pi K\left(G_{i}, i\right) .
$$

Let $E_{m}(\beta, g)$ be the fiber of the map

$$
K\left(Z_{q}, m\right) \stackrel{\beta}{\rightarrow} K(G) .
$$

For our purposes $q$ is either 0 or 2 . We will use $u_{m}$ to represent the characteristic class in $H^{m}\left(E_{m}\right)$. If $\alpha \beta=0$ is a relation on $m$ dimensional class then there is a class $v(\alpha) \in H^{*}\left(E_{m}\right)$ based on this relation. The triple $\left(E_{m}(\beta, q), u, v(\alpha)\right)$, thus, represents the universal example for a secondary operation defined on a class $a \in H^{m}\left(X, Z_{q}\right)$ with $\beta a=0$. Note also that $v(\alpha)$ could belong to different $E(\beta, q)$. For example $S q^{2} S q^{m-1}=0$ and $S q^{2} S q^{m-2}=0$ on $m-1 \mathrm{dim}$ integer classes so $v\left(S q^{2}\right) \in H^{*}\left(E\left(S_{q}{ }^{m-1}, 0\right)\right)$ and a different $v\left(S q^{2}\right) \in$ $H^{*}\left(E\left(S q^{m}, 0\right)\right)$. It is usually clear from the context.

The proof of 3.7 uses the following diagram

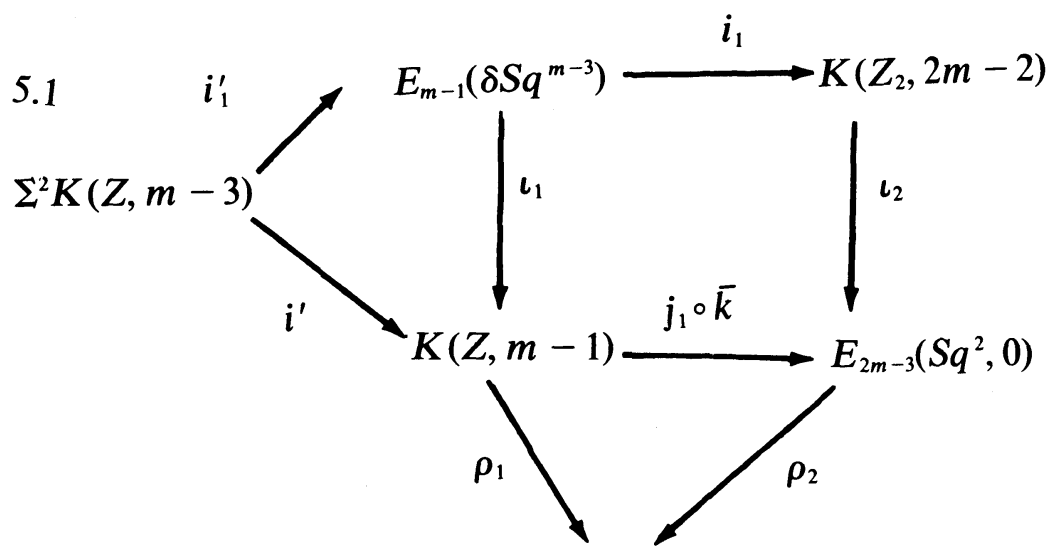

$K(Z, 2 m-3)$ 
The maps are defined as follows:

$$
j^{*} u_{m}=\delta u_{m-1} ; j * u_{2 m-3}^{*}=\delta S q^{m-3} ; k^{*} u_{m-1}=u_{m-1} \text {. }
$$

First we need to prove the existence of the diagram. The map $j$ is the one induced from the diagram

5.2

$$
E_{m}\left(S q^{m-1}, 0\right) \rightarrow K\left(Z_{2, m}\right) \stackrel{S q^{m-1}}{\longrightarrow} K\left(Z_{2}, 2 m-1\right)
$$

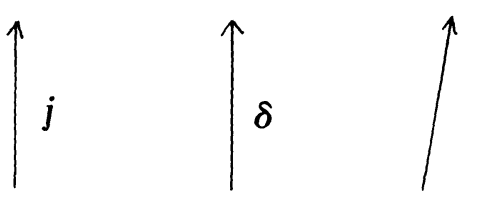

$$
E_{m-1}\left(S q^{m-1} S q^{1}, 2\right) \rightarrow K\left(Z_{2}, m-1\right) \text {. }
$$

The map $j_{1}$ is induced rom the diagram

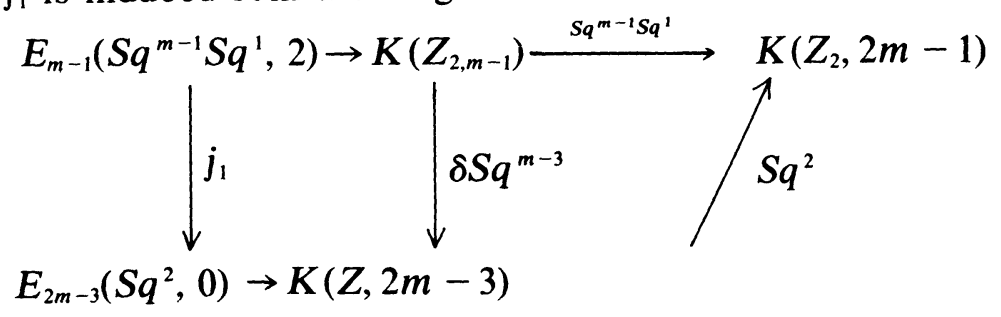

together with the observation that $S q^{2} \delta S q^{m-3}=S q^{m-1} S q^{1}$ on $m-1$ dimensional classes, $m \equiv 1(4)$.

The map $k$ exists because of the same relation. The map $i$ is the double adjoint and since $i^{*} \delta S q^{m-3} u=0$ the lifting $i$ exists.

Lemma 3.7 can be rephrased in this notation by the following.

Proposition 5.4. The class $v\left(S q^{2}\right)$ can be chosen so that $k^{*} j^{*} v\left(S q^{2}\right)=S q^{m-1} S q^{2} u_{m-1}$.

The first formula we need is

$$
j^{*} v\left(S q^{2}\right)=j_{i}^{*}\left(v\left(S q^{2}\right)+p_{i}^{*}(\gamma) .\right.
$$

This follows directly from diagram 5.2 and 5.3. Indeed, either diagram allows one to define an operation in $E_{m-1}\left(S q^{m-1} S q^{1}, 2\right)$ based on the relation $S q^{2} S q^{m-1} S q^{1}=0$. These two differ by some class in the base.

The second formula we need is $k^{*} j_{1}^{*}\left(v\left(S q^{2}\right)\right)=0$ modulo the indetermanancy, i.e., there is a choice of $k$ such that the formula is true. This implies that $k^{*} j^{*} v\left(S q^{2}\right)=k^{*} p_{i}^{*} \gamma$. We shall be finished when we evaluate

PROPOSITION 5.5. $\quad \gamma=S q^{m-1} S q^{2} u_{m-1}$. 
Proof. The map $K(Z, m-1) \underset{k^{\prime}}{\rightarrow} K\left(Z_{2}, m-1\right)$ lifts to a map $\bar{k}: K(Z, m-1) \rightarrow E_{m-1}\left(S q^{m-1} S q^{1}, 2\right)$. Clearly, $\bar{k}^{*} j^{*} v\left(S q^{2}\right)=0$. Thus, $\bar{k}^{*}{ }_{i}^{*} v\left(S q^{2}\right)=\gamma u_{m-1}$. Note that anything which is lost in $\gamma$ by evaluating it on an interger class is part of the ambiguity in defining $v \in$ $H^{2 m}\left(E_{m}\left(S q^{m-1}, 0\right)\right)$.

We have the following diagram

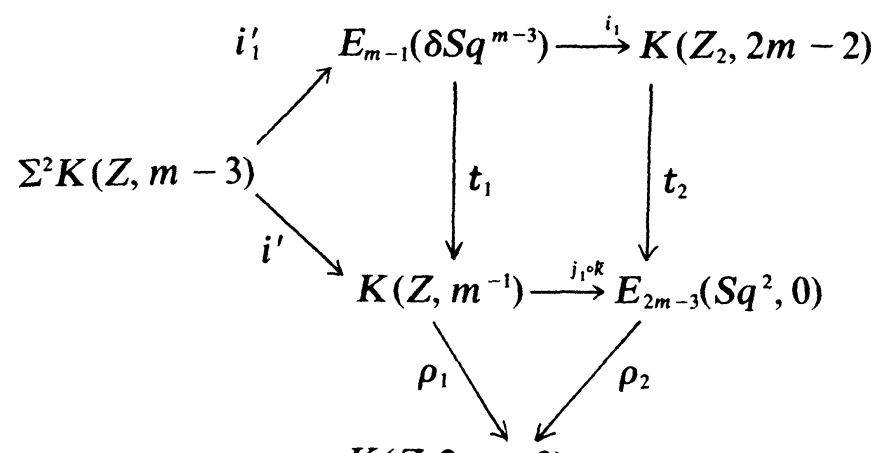

$K(Z, 2 m-3)$

A direct check of the appropriate exact sequence shows that

$$
i_{i}^{*} \kappa_{2 m-2}=v\left(S q^{2}\right) .
$$

It follows from [4] that $i_{1}^{\prime *}\left(v\left(S q^{2}\right)\right)=\sigma^{2}\left(\kappa \cup S q^{2} \kappa\right)$. Since $\iota_{2}^{*} v\left(S q^{2}\right)=$ $S q^{2} \kappa_{2 m-2}$, we see that

$$
\iota *\left(j_{1} \circ \bar{k}\right)^{*} v\left(S q^{2}\right)=S q^{2}\left[v\left(S q^{2}\right)\right]
$$

Since $\operatorname{ker} i^{\prime *}=\operatorname{ker} \iota_{1}^{*}$ in this dimension we have

$$
\begin{aligned}
i^{\prime *}\left(j_{1} \circ \bar{k}\right) * v\left(S q^{2}\right) & =S q^{2}\left(\sigma^{2}\left(\kappa \cup S q^{2} \kappa\right)\right. \\
& =S q^{m-1} S q^{2}\left(\sigma^{2} \kappa\right)
\end{aligned}
$$

Thus, $\left(j_{1} \circ \bar{k}\right)^{*} v\left(S q^{2}\right)=S q^{m-1} S q^{2} \kappa_{m-1}$. This proves the proposition and completes the proof of the theorem.

It is interesting to note that the above argument proves the following theorem.

THEOREM 5.6. In $H^{*}(K(Z, m-1)), m \equiv 1(4), \quad \varphi_{1,1}\left(\delta S q^{m-4}\right)=$ $S q^{m-1} S q^{2}$ mod the indeterminancy where $\varphi_{1,1}$ is the secondary operation defined on integer classes based on $S q^{2} S q^{2}=0$. 


\section{REFERENCES}

1. M. Atiyah, Vector fields on manifolds, Arberlsgemeinschaft fur Fosschuny des Landes Nordrhein Westfalen, Hept. 200.

2. E. Brown and F. Peterson, Whitehead products and chomology operations. Quart. J. Math., 15 (1964), 116-120.

3. G. Lusztig, J. Milnor, and F. Peterson, Semi-characteristic and cobordism, Topology, 8 (1969), 357-360.

4. M. Mahowald and F. Peterson, Secondary operations on the Thom class, Topology, 2 (1964), 367-377.

5. E. Thomas, The index of a tangent 2-field, Comment. Math. Helv., 42 (1967), 86-110.

Received December 24, 1973. This work was supported in part by the NSF GP 25335.

NORTHWESTERN UNIVERSITY 


\section{PACIFIC JOURNAL OF MATHEMATICS}

\section{EDITORS}

RICHARD ARENS (Managing Editor)

University of California

Los Angeles, California 90024

\section{J. DugundJI}

Department of Mathematics University of Southern California Los Angeles, California 90007

D. Gilbarg and J. Milgram

Stanford University

Stanford, California 94305

\section{ASSOCIATE EDITORS}
E. F. BECKENBACH
B. H. NeumanN
F. WoLF
K. YoshiDA

\section{SUPPORTING INSTITUTIONS}

\author{
UNIVERSITY OF BRITISH COLUMBIA \\ CALIFORNIA INSTITUTE OF TECHNOLOGY \\ UNIVERSITY OF CALIFORNIA \\ MONTANA STATE UNIVERSITY \\ UNIVERSITY OF NEVADA \\ NEW MEXICO STATE UNIVERSITY \\ OREGON STATE UNIVERSITY \\ UNIVERSITY OF OREGON \\ OSAKA UNIVERSITY
}

\author{
UNIVERSITY OF SOUTHERN CALIFORNIA \\ STANFORD UNIVERSITY \\ UNIVERSITY OF TOKYO \\ UNIVERSITY OF UTAH \\ WASHINGTON STATE UNIVERSITY \\ UNIVERSITY OF WASHINGTON \\ AMERICAN MATHEMATICAL SOCIETY
}

The Supporting Institutions listed above contribute to the cost of publication of this Journal, but they are not owners or publishers and have no responsibility for its contents or policies.

Mathematical papers intended for publication in the Pacific Journal of Mathematics should be in typed form or offset-reproduced (not dittoed), double spaced with large margins. Underline Greek letters in red, German in green, and script in blue. The first paragraph or two must be capable of being used separately as a synopsis of the entire paper. Items of the bibliography should not be cited there unless absolutely necessary, in which case they must be identified by author and Journal, rather than by item number. Manuscripts, in duplicate, may be sent to any one of the four editors. Please classify according to the scheme of Math. Reviews, Index to Vol. 39. All other communications should be addressed to the managing editor, or Elaine Barth, University of California, Los Angeles, California, 90024.

100 reprints are provided free for each article, only if page charges have been substantially paid. Additional copies may be obtained at cost in multiples of 50 .

The Pacific Journal of Mathematics is issued monthly as of January 1966. Regular subscription rate: $\$ 72.00$ a year (6 Vols., 12 issues). Special rate: $\$ 36.00$ a year to individual members of supporting institutions.

Subscriptions, orders for back numbers, and changes of address should be sent to Pacific Journal of Mathematics, 103 Highland Boulevard, Berkeley, California, 94708.

PUBLISHED BY PACIFIC JOURNAL OF MATHEMATICS, A NON-PROFIT CORPORATION Printed at Jerusalem Academic Press, POB 2390, Jerusalem, Israel.

$$
\begin{gathered}
\text { Copyright (C) } 1975 \text { Pacific Journal of Mathematics } \\
\text { All Rights Reserved }
\end{gathered}
$$




\section{Pacific Journal of Mathematics}

\section{Vol. 58, No. $2 \quad$ April, 1975}

Zvi Artstein and John Allen Burns, Integration of compact set-valued functions . . . . . . . . . 297

Mark Benard, Characters and Schur indices of the unitary reflection group $[321]^{3} \ldots \ldots \ldots . .309$

Simeon M. Berman, A new characterization of characteristic functions of absolutely continuous

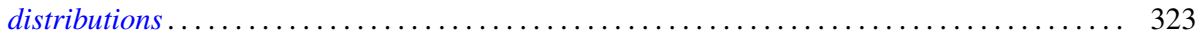

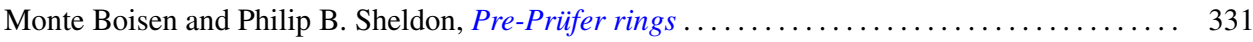

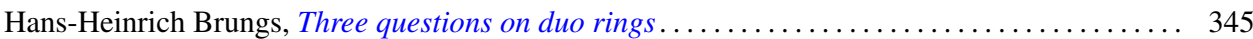

Iracema M. Bund, Birnbaum-Orlicz spaces of functions on groups................. 351

John D. Elwin and Donald R. Short, Branched immersions between 2-manifolds of higher

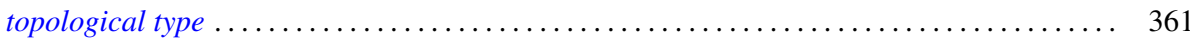

Eric Friedlander, Extension functions for rank 2, torsion free abelian groups . .......... 371

Jon Froemke and Robert Willis Quackenbush, The spectrum of an equational class of

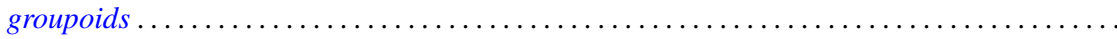

Barry J. Gardner, Radicals of supplementary semilattice sums of associative rings ...........

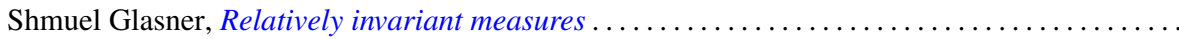

George Rudolph Gordh, Jr. and Sibe Mardesic, Characterizing local connectedness in inverse

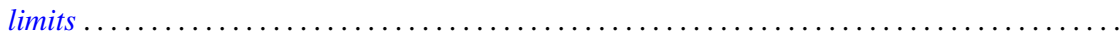

Siegfried Graf, On the existence of strong liftings in second countable topological spaces......

Stanley P. Gudder and D. Strawther, Orthogonally additive and orthogonally increasing

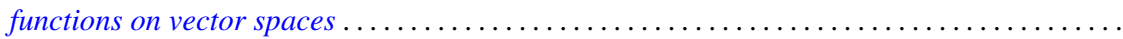

Darald Joe Hartfiel and Carlton James Maxson, A characterization of the maximal monoids and

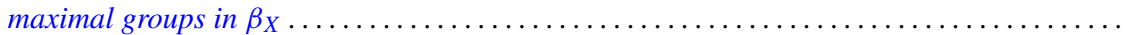

Robert E. Hartwig and S. Brent Morris, The universal flip matrix and the generalized faro-shuffle. .

William Emery Haver, Mappings between ANRs that are fine homotopy equivalences. .

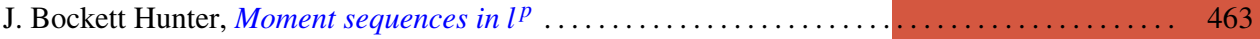

Barbara Jeffcott and William Thomas Spears, Semimodularity in the completion of a poset.... 467

Jerry Alan Johnson, A note on Banach spaces of Lipschitz functions . . . . . . . . . . . . 475

David W. Jonah and Bertram Manuel Schreiber, Transitive affine transformations on

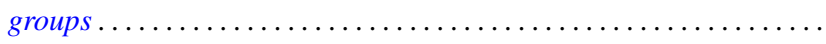

Karsten Juul, Some three-point subset properties connected with Menger's characterization of

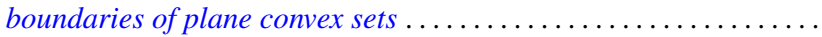

Ronald Brian Kirk, The Haar integral via non-standard analysis . . . . . . . . . . . . . 517

Justin Thomas Lloyd and William Smiley, On the group of permutations with countable

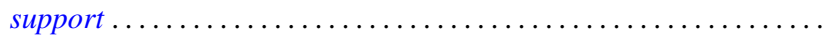

Erwin Lutwak, Dual mixed volumes .................................. 531

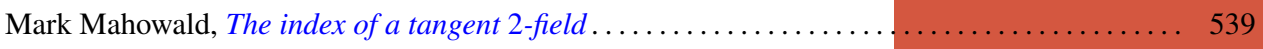

Keith Miller, Logarithmic convexity results for holomorphic semigroups . . . . . . . . . . . . 549

Paul Milnes, Extension of continuous functions on topological semigroups . . . . . . . . . . 553

Kenneth Clayton Pietz, Cauchy transforms and characteristic functions ................ 563

James Ted Rogers Jr., Whitney continua in the hyperspace $C(X) \ldots \ldots \ldots \ldots \ldots \ldots \ldots \ldots .569$

Jean-Marie G. Rolin, The inverse of a continuous additive functional . . . . . . . . . . . . 585

William Henry Ruckle, Absolutely divergent series and isomorphism of subspaces . ........ 605

Rolf Schneider, A measure of convexity for compact sets . ..................... 617

Alan Henry Schoenfeld, Continous measure-preserving maps onto Peano spaces .......... 627

V. Merriline Smith, Strongly superficial elements .......................... 643

Roger P. Ware, A note on quadratic forms over Pythagorean fields . . . . . . . . . . . . . . 651

Roger Allen Wiegand and Sylvia Wiegand, Finitely generated modules over Bezout rings . . . . 655

Martin Ziegler, A counterexample in the theory of definable automorphisms . . . . . . . . . 665 